\title{
Pratiques
}

Linguistique, littérature, didactique

149-150 | 2011

Didactique du français (2)

\section{L'énonciation des brouillons et la question du sujet scolaire}

Catherine Boré

\section{(2) OpenEdition}

Journals

Édition électronique

URL : http://journals.openedition.org/pratiques/1709

DOI : 10.4000/pratiques.1709

ISSN : 2425-2042

Éditeur

Centre de recherche sur les médiations (CREM)

Édition imprimée

Date de publication : 15 juin 2011

Pagination : 71-90

Référence électronique

Catherine Boré, «L'énonciation des brouillons et la question du sujet scolaire », Pratiques [En ligne], 149-150 | 2011, mis en ligne le 16 juin 2014, consulté le 01 mai 2019. URL : http://

journals.openedition.org/pratiques/1709; DOI : 10.4000/pratiques.1709

(c) Tous droits réservés 


\title{
L'énonciation des brouillons et la question du sujet scolaire
}

\author{
Catherine Boré \\ Université de Cergy-Pontoise-IUFM \\ EMA EA 4507 \& Modyco UMR 7114
}

\section{Introduction}

Avant qu'elle ne devienne une question pour la classe, l'étude des brouillons était réservée aux manuscrits littéraires, et le brouillon d'écolier jeté dans la « poubelle de la classe » (Fabre, $\left.1982^{(1)}\right)$. Apparue d'abord au primaire ${ }^{(2)}$ avec le développement de la didactique de l'écriture, sous l'influence de travaux de Claudine Fabre, l'installation des brouillons dans les programmes comme objet didactique, souvent confondue et prolongée avec celle de réécriture, a introduit une série de questions parmi lesquelles l'énonciation et la présence du sujet, la question de l'auteur, du scripteur, du style.

Différents articles de synthèse (parmi de nombreuses références ${ }^{(3)}$, Fabre 2004 et plus récemment Garcia-Debanc Sonia Branca 2007, Doquet-Lacoste 2008), ont retracé le double héritage théorique - génétique textuelle et théorie de l'énonciation - qui a permis le développement de l'objet d'étude "brouillon » en didactique de l'écriture ${ }^{(4)}$. Mais au fond quelle part de ces théories se trouve au juste concernée dans la pratique didactique ? Une telle question peut concerner la notion un peu floue « d'énonciation d'un sujet», dont la proximité avec les notions d'enfant écrivain (Clanché, 1988), de sujet écrivant (Lafont-Terranova, 2009), d'élève-auteur (Plane, 2005) - ou plus globalement, de sujet didactique (Reuter, Delcambre, 2007), manifeste la difficulté qu'il y a à qualifier l'énonciation d'un sujet scripteur

(1) Fabre, Claudine (1982): «Dans la poubelle de la classe : subjectivité et jeux de langage »La linguistique volume 18, fascicule 2, pp. 99-113.

(2) Maîtrise de la langue à l'école, 1992.

(3) Impossible de citer tous les auteurs qui, en tant que spécialistes de l'écriture, ont pu faire des synthèses : Petitjean 1999, Barré de Miniac 2000, Reuter et Delcambre 2002, Plane $2003 .$.

(4) Je laisse volontairement de côté les développements de psychologie cognitive qui occupent une place importante dans les recherches sur la genèse de l'écriture - notamment à Poitiers les travaux du GDR 2657 «Production verbale écrite» (D. Alamargot, dir.), pour m'en tenir au domaine linguistique. 
(Bishop, 2007, Tauveron, 2007) dans le cadre de l'école : comment en effet désigner l'enfant qui écrit à l'école ?

Je tâcherai d'abord de montrer quel(s) sens de « sujet» et d'《énonciation » la didactique a privilégiés par rapport aux théories de référence, puis je proposerai en deux temps une définition du sujet appuyée sur différents aspects du dialogisme bakhtinien en montrant la fécondité de cette approche à partir d'exemples d'écriture scolaire.

\section{Les brouillons en didactique de l'écriture : usages d'une théorie de l'énonciation}

\section{Subjectivité et énonciation : le sujet en questions}

Si l'on convient que le sujet de l'écriture n'est ni le sujet empirique/biographique ni le sujet psychologique ${ }^{(5)}$, on pourrait proposer un sujet scolaire de l'écriture pour accentuer ce que peut être l'appropriation de la langue dans sa particularité institutionnelle.

Il faut rendre à D. Bucheton (1995) la priorité du concept de sujet scolaire évoqué dans son livre si personnel et l'un des premiers ${ }^{(6)}$ à s'être intéressé à l'écriture du point de vue d'un sujet scolaire. Sa conclusion est encore d'actualité. Je la cite car elle ouvrait la possibilité d'une réflexion nouvelle sur le scripteur mué en sujet spécifiquement « scolaire » :

« La didactique du français, didactique de la parole, aide le sujet à construire son rapport particulier au monde, à lui-même, aux textes, aux paroles des hommes de ce monde, à leur culture polymorphe, polytechnique, polyethnique...Son rôle est d'aider le sujet à s'approprier les outils langagiers, les modes de pensée qui lui permettront d'occuper par la parole une place singulière dans ce monde. Mais, dire le monde, se dire dans le monde, nécessite l'apprentissage de l'écoute des autres. Cette singularité du sujet que le langage instaure, se construit par le partage de la parole écrite, orale. Tel me semble être le projet fondateur de la didactique du français, tels me semblent être les principes très concrets, devant servir de soubassement aux situations d'enseignement-apprentissage.

$[\ldots]$

C'est à ce sujet scolaire (certes socialement, culturellement, affectivement et psychologiquement constitué par sa vie extérieure à l'école) que l'enseignement s'adresse. C'est ce sujet scolaire-là qu'il est de notre responsabilité d'enseignant de former, à qui nous devons offrir et structurer un espace pour qu'il y puisse exercer sa pensée et sa parole. » (Bucheton, 1995 : 289-290)

(5) L'étude de Bishop (2007) fait une lecture historique des textes officiels de français de l'école élémentaire concernant la manière dont le sujet scolaire de l'écriture s'est constitué depuis 1882 ; elle note que l'acte de naissance d'un sujet individuel à l'école date des instructions de 1972. Cependant l'élève apparaît sujet bien avant, sous des formes diverses : comme sujet «moral » de la III ${ }^{\mathrm{e}}$ République ; comme individu doté d'une histoire personnelle à la fin des années 1960 ; et comme un sujet cognitif, tel qu'il se dessine depuis la fin $\mathrm{du} \mathrm{XX}^{\mathrm{e}}$ siècle. Mais l'approche ne prend pas en compte centralement le rapport à la langue (voir ci-après mon développement sur le rapport entre société et langue).

(6) Les auteurs sont nombreux à avoir parlé de l'écriture des élèves comme sujets : je n'oublie pas, bien évidemment, les ouvrages de Pierre Clanché, Clairelise Bonnet et al., Claudine Garcia-Debanc, Mireille Froment, 1988, Claudine Fabre, 1990, etc., certains cités plus haut. 
Ce sujet me semble différent du « sujet didactique » de l'écriture défini par Delcambre \& Reuter (Delcambre, 2007) dans une autre acception, à dominante sociologique. Il est issu d'images construites, de représentations qu'il donne de luimême, que ces représentations s'appuient sur des interviews faites par le chercheur auprès de l'enquêté ou des retours réflexifs en classe plus ou moins guidés. L'image du scripteur se déduit aussi bien d'enquêtes formelles mais il faut se contenter des connaissances déclaratives qu'il a bien voulu livrer.

L'élaboration de ce sujet didactique est un construit dont I. Delcambre emprunte à Y. Reuter la définition suivante, appliquée [aux] :

«... acteurs, en tant qu'ils sont constitués par des relations d'enseignement et d'apprentissage, institutionnalisées, à des objets de savoir, référés à des disciplines $\gg{ }^{(7)}$.

Cette définition a le mérite de poser la spécificité d'un sujet institutionnel, le rapportant aux principaux objets qui le fondent : relation aux savoirs, aux disciplines, à travers les conduites cognitives d'enseignement-apprentissage.

Pourtant ce sujet didactique apparaît comme une construction " totale »: somme descriptive, on peut craindre qu'elle ne mette affects, inconscient, origines, goûts, etc. de l'élève au service d'une causalité expliquant son comportement de scripteur. La modélisation proposée ou envisagée part de l'option que l'institution Ecole absorbe et détermine entièrement le sujet didactique.

Face à ce sujet sociologique, on rappellera la position de Benveniste d'une netteté extrême dans l'article «Sémiologie de la langue » ${ }^{(8)}$. Pour Benveniste, la langue est l'interprétant ultime. Benveniste dégage la langue en tant que système sémiotique d'autres systèmes censés les inclure, comme les systèmes sociaux. Il récuse l'idée que la langue en usage dans toute société soit englobée par celle-ci suivant une analyse de la partie et du tout :

«Si l'on s'interroge par exemple sur la situation respective de la langue et de la société - thème à débats incessants- et sur leur mode de dépendance mutuelle, le sociologue, et probablement quiconque envisage la question en termes dimensionnels, observera que la langue fonctionne à l'intérieur de la société, qui l'englobe ; il décidera donc que la société est le tout, et la langue, la partie. Mais la considération sémiologique inverse ce rapport, car seule la langue permet la société. La langue constitue ce qui tient ensemble les hommes, le fondement de tous les rapports qui à leur tour fondent la société. On pourra dire alors que c'est la langue qui contient la société. Ainsi la relation d'interprétance, qui est sémiotique, va à l'inverse de la relation d'emboîtement, qui est sociologique. Celle-ci objectivant les dépendances externes, réifie pareillement le langage et la société, tandis que celle-là les met en dépendance mutuelle selon leur capacité de sémiotisation » ${ }^{(9)}$.

Avec Benveniste, s'affirme une analyse qui ne laisse pas de place à une définition sociologique du sujet, quelles qu'en soient les nuances (Lahire $v s$ Bourdieu) :

(7) Y. Reuter, Dictionnaire des concepts fondamentaux des didactiques Bruxelles, De Boeck (2007: 91) cité par Delcambre ibid. p. 39.

(8) Benveniste, E. 1969, «Sémiologie de la langue» dans Semiotica La Haye, Mouton \&Co, repris dans Problèmes de Linguistique Générale Tome 2, chapitre 3, pp. 43-66, TEL Gallimard.

(9) Ibid. p. 62 . 
une conception " explicative » du sujet scolaire, tout comme une conception statistique ou quantitative méconnaît que la pluralité du sujet réside dans son rapport à la langue.

Toute la place est donc laissée à l'énonciation qui s'effectue dans le cadre scolaire et c'est à ce niveau qu'elle doit être observée.

Je développerai donc une approche du sujet scolaire à partir de ce qui fait de lui un énonciateur, c'est-à-dire par la mise en usage qu'il fait de la langue, usage tout à la fois commun et idiolectal.

Les brouillons sont les lieux où s'élabore ce sujet, dont l'espace d'énonciation est tout à fait original.

\subsection{Rappel d'une problématique ancienne : les brouillons ou la figuration d'une énonciation}

Comme je l'ai rappelé en introduction, «l'enfant écrivain » encore appelé « écrivant», n'est devenu « scripteur » qu'avec la découverte que ce qui était laissé dans la " poubelle de la classe » pouvait porter témoignage comme trace d'une activité plutôt que d'un acte.

Cet événement fut et reste considérable à la fois pour la linguistique, la cognition, la littérature et la didactique.

En didactique, l'accent mis par les programmes sur l'énonciation au brouillon a plutôt resserré l'attention sur le geste de production que sur les formes nécessairement dialogiques qu'implique l'énonciation écrite.

C'est sans doute la raison pour laquelle on s'est beaucoup préoccupé des facteurs favorisant la réflexivité du langage en travaillant la co-élaboration préalable et continue de l'écriture par la parole conjointe des élèves et du maître, par le travail collaboratif des élèves dans les tâches d'écriture, et parallèlement aux brouillons, l'élaboration du texte par les «écrits intermédiaires » ${ }^{(10)}$, écrits de travail ou carnets de lecture/écriture ${ }^{(11)}$. De ce fait, le travail de l'énonciation se voit déplacé, dans un geste d'élargissement souvent de nature épi-linguistique.

Les travaux engagés à la suite de Claudine Fabre, sur le matériau «non noble» des brouillons d'élèves, ont tenté de conjoindre étude de la langue en mouvement, activité d'apprentissage d'un scripteur, élaboration d'une activité scripturale investie par un sujet. C'est le travail du brouillon, en effet, qui - beaucoup plus que la thématique de l'écrit -, qu'elle soit fictionnelle ou factuelle, a transformé l'élève et l'enfant créatif/créateur en sujet de l'écriture.

Pour expliquer l'importance des travaux de la génétique textuelle consacrés aux brouillons, sans doute faut-il aller un peu plus loin que la réception assez réductrice qui en a été faite dans le cadre scolaire.

Dès le début des années 1990, en effet, on trouve dans les IO des recommandations visant à relire son brouillon et à utiliser pour améliorer son écrit les opéra-

(10) Voir par exemple Bucheton, D., \& Chabanne, J.-C., (2002) Parler et écrire pour apprendre, penser et se construire PUF, collection «L'éducateur».

(11) Claire Doquet-Lacoste (2007) : «Promenade dans les bois du littéraire et d'ailleurs : les carnets des élèves en classe », 213-222 in: Dufays, J.-L. (dir.) : Enseigner et apprendre la littérature aujourd'hui, pour quoi faire? Sens, utilité, évaluation, Presses Universitaires de Louvain, collection « Recherches en formation des enseignants et en didactique ». 
tions de réécriture, de même que se développent, sous l'influence des modèles cognitifs nord-américains, des modèles de production d'écrit qui présupposent un contrôle de la part du scripteur postérieurement à son écrit. Tout ceci est bien connu, et ne sera pas développé. Cependant l'apport fondamental des études génétiques a consisté à se centrer sur l'activité d'énonciation du sujet plutôt que sur le résultat de l'énonciation, ce qui explique la difficulté de la démarche en général et encore plus dans le contexte solaire.

Dans un numéro de Langages ${ }^{(12)}$, J.-L. Lebrave ${ }^{(13)}$ entreprenait de distinguer le brouillon du «texte», définissant minimalement le premier comme «du texte qui contient des substitutions chronologiquement orientées. " (Lebrave, 1983 : 13).

Il mettait ainsi en évidence le travail de reconstitution qui aboutit à envisager la production du brouillon "comme une séquence d'événements qui se succèdent dans le temps. » (1983: 15).

La rature est un des éléments capables de figurer la marque du temps : l'écoulement arrêté du flux d'écriture par cette matérialisation qui fait retour sur ellemême. D'autres éléments attestent de cet écoulement du temps de l'écriture : les blancs, les phrases inachevées, les nouveaux départs sur de nouveaux supports : nouveau paragraphe, nouveau texte, nouvelle page. Allant plus loin, il analysait l'activité de lecture des brouillons et manuscrits comme une glose qui "transforme les objets spatiaux que sont les fragments d'écriture en objets textuels ordonnés le uns par rapport aux autres [...] » (ibid.).

Arrêtons-nous cependant sur le sens d'énonciation qui vient d'être envisagé : il s'agit d'un sens restrictif qui fait de l'énonciation le synonyme de l'activité d'énoncer, activité différente selon qu'on se place du point de vue du « producteur » ou du lecteur de l'énoncé.

A l'école, les brouillons sont souvent considérés comme des supports, comme une série d'objets discrets et substituables : telle est devenue la mécanique des « trois états » successifs matérialisés par des feuilles différentes, sur lesquelles le jeune scripteur concentrerait son activité métalinguistique, bien peu visible alors, tant elle semble la reprise à neuf d'une nouvelle tâche d'écriture.

Mais pour qui veut bien relier ensemble cette série d'écrits orientés dans le temps, les brouillons apparaissent comme les produits émergés d'une énonciation continue qui s'élabore sous nos yeux. Et l'on peut dire à ce titre que le brouillon révolutionne la segmentation des unités du texte, en donnant à voir l'élaboration d'un mot, d'un syntagme ou d'un ensemble de lettres, d'abord non signifiants puis peu à peu déployés.

Simultanément s'impose un constat : si l'énonciation est continue parce qu'elle est inévitablement orientée dans le temps, elle est aussi piétinement, voire dédoublement. Faisant retour sur elle-même, l'énonciation réitère (recopie du même, j'y reviendrai) ou fait arrêt sur les mots : c'est le sens des «boucles énonciatives » dont J. Authier-Revuz (1995) a déployé toute la complexité dans le discours en train de se faire.

L'énonciation des brouillons est ainsi, dans ce premier sens, une dialectique du continu et du discontinu.

(12) Langages, $\mathrm{n}^{\circ} 69$ (1983).

(13) Dans son article «Lecture et analyse des brouillons» : 11-23. 


\subsection{L'enracinement chez Benveniste : l'énonciation d'un sujet}

Benveniste dans sa formule fameuse «L'énonciation est cette mise en fonctionnement de la langue par un acte individuel d'utilisation " ${ }^{(14)}$ relève trois grands critères dans le procès d'énonciation : la réalisation vocale de la langue, le mécanisme de production de l'énonciation, qui vise à décrire la manière dont le sens se forme en mots, et la définition de l'énonciation dans le cadre formel de sa réalisation. C'est ce dernier point qui constitue l'essentiel de l'article contenu dans ces lignes :

« Nous tentons d'esquisser, à l'intérieur de la langue, les caractères formels de l'énonciation à partir de la manifestation individuelle qu' elle actualise. » ${ }^{(15)}$

Ce projet peut passer pour paradoxal : il ne s'agit de rien de moins que, partant de la diversité infinie et contingente du discours en acte, d'en marquer les éléments constants, inhérents, et proprement formels, langue et discours apparaissant alternativement à l'analyse.

«En tant que réalisation individuelle, l'énonciation peut se définir, par rapport à la langue, comme un procès d'appropriation. Le locuteur s'approprie l'appareil formel de la langue et il énonce sa position de locuteur par des indices spécifiques, d'une part, et au moyen de procédés accessoires, de l'autre. » ${ }^{(16)}$

Benveniste envisage ainsi langue et discours comme deux ordres vus alternativement et complémentairement.

\subsubsection{L'acte d'énonciation n'est pas l'acte de production}

L'analyse de Benveniste implique qu'à l'instant où se crée l'énonciation, se construise aussi le sujet, qui ne préexiste donc pas à son acte. Le sujet est à l'œuvre dans la parole « actuelle» et non pas comme entité préexistante.

C'est ce qui explique que l'acte d'énonciation ne soit pas l'acte de production: en effet, parler d'acte de production c'est mettre l'accent sur l'activité cognitive du locuteur, objectivée et isolée. C'est aussi considérer l'activité du seul locuteur. $\underline{\text { Benveniste insiste au contraire sur l'activité double du locuteur et de l'allocutaire }}$ qui fait de l'acte d'énonciation un « dialogue ». On oublie en effet de citer la suite de la phrase célèbre ci-dessus :

« Mais immédiatement, dès qu'il se déclare locuteur et assume la langue, il implante l'autre en face de lui, quel que soit le degré de présence qu'il attribue à cet autre. Toute énonciation est, explicite ou implicite, une allocution, elle postule un allocutaire. » ${ }^{(17)}$

Ainsi, à partir d'une première relation dialectique liant langue et discours dont le produit est l'énonciation, s'enclenche un caractère formel premier et fonda-

(14) Benveniste, « Problèmes de linguistique générale » [1970, 1974], La communication, chapitre 3 «La sémiologie de la langue » Tome II, TEL Gallimard, p. 80.

(15) Ibid., p. 81.

(16) Ibid., p. 82.

(17) Ibid., p. 82. 
mental : la «virtualité » de dialogue impliquée par cet acte. L'énonciation en acte implique donc de façon nécessaire une relation, qu'elle soit virtuelle ou actuelle. Benveniste commente longuement les formes linguistiques impliquées, depuis les modalités d'énonciation et d'énoncé jusqu'à l'accentuation de la relation discursive au partenaire ${ }^{(18)}$.

« Comme forme de discours, l'énonciation pose deux « figures » également nécessaires, l'une source, l'autre but de l'énonciation. C'est la structure du dialogue. Deux figures en position de partenaires sont alternativement protagonistes de l'énonciation. Ce cadre est donné avec la définition de l'énonciation. » ${ }^{(19)}$

L'analyse de Benveniste, faire du dialogue la structure inhérente de l'énonciation, en révèle en même temps le caractère formel : la langue convertie en discours par l'énonciation se maintient sous la structure des oppositions formelles comme je/tu vs il.

Ainsi la notion de sujet de l'énonciation (écrite) est-elle une notion dynamique qui met en jeu le locuteur (scripteur) tel que le (re)construisent les mots dont il se sert pour se situer dans son espace-temps. Benveniste en associant cet acte à des traces linguistiques a du même coup impliqué la nécessité d'un allocutaire. En effet, comme le remarque Y. Erard ${ }^{(20)}$ dans son analyse de l'énonciation chez Benveniste, ces traces n'ont de sens que décodées par "l'interaction interprétative du locuteur et du co-locuteur », et non pas seulement comme centre de référence interne : l'énonciation n'est effective qu'à la condition de cette double activité. Le sujet, incarné par son rapport au temps et au lieu, est aussi reconnu comme tel par son interprète.

Benveniste a insisté en des termes qui font écho de façon surprenante aux théories du dialogue (voir ci-après), dans un passage célèbre de l'article de Langages de 1970 L'appareil formel de l'énonciation, sur la généralisation du dialogue, sur ce qu'on pourrait appeler sa racine anthropologique :

«[...] le monologue procède bien de l'énonciation. Il doit être posé, malgré l'apparence, comme une variété du dialogue, structure fondamentale. Le "monologue" est un dialogue intériorisé formulé en "langage intérieur" entre un moi locuteur et un moi écouteur. Parfois le moi locuteur est seul à parler; le moi écouteur reste néanmoins présent ; sa présence est nécessaire et suffisante pour rendre signifiante l'énonciation du moi locuteur. Parfois aussi le moi écouteur intervient par une objection, une question, un doute, une insulte. La forme linguistique que prend cette intervention diffère selon les idiomes, mais c'est toujours une forme personnelle. Tantôt le moi écouteur se substitue au moi locuteur et s'énonce donc comme "première personne"; ainsi en français où le monologue sera coupé de remarques ou d'injonctions telles que : "Non je suis idiot j'ai oublié de lui dire que..." tantôt le moi écouteur interpelle à la "deuxième personne" le moi locuteur : "Non tu n'aurais pas dû lui dire que...". »" ${ }^{(21)}$

(18) Ibid., p. 85.

(19) Ibid.

(20) Erard, Y. (1998) : De l'énonciation à l'énaction. L'inscription corporelle de la langue Cahiers de 1'ILSL, $\mathrm{N}^{\circ}$ 11, 91-121, pp. 94-96.

(21) Voir le chapitre V des Problèmes de Linguistique Générale, TEL Gallimard, tome II, pp. 85-86. 
Ainsi pour Benveniste la subjectivité est inscrite dans le discours par l'utilisation de « je ».

Mais dire « je » recouvre plusieurs significations : outre le fait qu'il existe un «je» générique particulièrement présent à l'école ${ }^{(22)}$, dire « je » est tout autant : asserter ${ }^{(23)}$, décrire un «soi » au passé par le marqueur «je», représenter «je s'énonçant " par le biais du discours rapporté comme le remarque Benveniste, etc., toutes acceptions qui font des actes différents. En quoi, alors, dire « je » serait-il une manifestation univoque de la subjectivité ? ${ }^{(24)}$ Et corollairement, le sujet ne peutil s'énoncer autrement qu'en disant « je » ${ }^{(25)}$

Ces questions, qui ont d'ailleurs été en partie évoquées par Benveniste luimême, sont légitimes (aussi bien n'est-ce pas le sens du marqueur « je » qui est ici en cause), mais elles ne peuvent restreindre la portée de la découverte de Benveniste qui réside dans l'incarnation de l'homme dans la langue : sujet par la parole qui l'amarre à la relation et simultanément dénote son incarnation dans le temps et le lieu.

La double caractéristique d'être homme par la parole et par la relation fait de lui un "être dialogué [...] amarré à un réel de langage " ${ }^{(26)}$. C'est cet aspect que je voudrais développer maintenant, en appui sur l'interprétation bakhtinienne du dialogisme.

(22) Comme dans les listes des « droits et devoirs » que l'on trouve parfois affichés en classe : « je lève le doigt pour poser une question »; « je n'interromps pas un camarade quand il parle »; «j'ai bien relu mon texte », etc.

(23) Le sens d'assertion d'un acte de langage est une des acceptions de sujet (inévitablement) exprimé par le marqueur «je », que donne J.-C. Coquet 1998, dans La quête du sens, Presses universitaires de France, collection «Formes sémiotiques », Paris, p. 248 :

"Pour éviter l'extension abusive de la notion de sujet, dont on relève des exemples même chez Merleau-Ponty, l'analyste est donc conduit à proposer une classe actancielle construite sur l'exclusion du jugement, celle du non-sujet, où le préfixe non-marque l'absence, comme dans l'opposition privative.

En me plaçant sur un plan général, je dirai qu'il faut poser la bivalence de l'actant : sous l'un de ses formes, celle du sujet, il asserte sa parole et ses actes; sous l'autre, il se contente de le prédiquer. Aussi bien, la prédication suffit-elle à caractériser le non-sujet alors que prédiquer et asserter, combinés en un seul sujet, caractérisent le sujet. Pour mettre en scène son univers, l'instance énonçante prédique ; pour la prendre à son compte, elle asserte." "

(24) Ana Ono (2007) dans son étude sur l'énonciation chez Benveniste montre qu'il s'agit d'une acception particulière d'énonciation. En contrepoint et complémentairement, il apparaît que, pour Benveniste, la subjectivité est tout autant dans la langue que dans le discours; mais elle y est présente virtuellement, « imprégnant le système linguistique tout entier » (Ono, 2007 : 141) au point que la subjectivité doit être comprise non pas seulement par des marques spécifiques (déictiques par exemple) mais « comme le sujet en tant que langue » pour reprendre les termes dont se sert un commentateur de Benveniste cité par A. Ono : le sujet n'est pas séparé du langage, pas plus que l'acte de discours n'est «jeté en-dehors de la langue »(Ono, $2007: 142)$. Le sujet, en ce sens, c'est-à-dire imprégnant la langue, est repérable dans la structure idiomatique des langues : par exemple dans la structure schématique des prépositions latines pro et prae qui dénotent une position externe ou interne du sujet du procès. C'est alors ici de « la subjectivité dans le langage »(C. Kerbrat-Orecchioni) qu'il s'agit.

(25) Ce problème philosophique est l'objet du livre de S. Chauvier (2001), qui conclut que la subjectivité est identique à l'aptitude à dire « je ».

(26) L'expression est de Ph. Schepens, 1999: 165. 


\section{Le cadre du dialogisme}

\subsection{Un bref rappel}

Le dialogisme bakhtinien prend naissance à la fin des années 1920, alors que règne une grande effervescence intellectuelle en Russie autour de la notion de dialogue. Linguistes, psychologues et philosophes s'y intéressent et parmi eux Jakubinski (1892-1945), qui développe la thèse extrême que le dialogue est la forme première «naturelle » de la communication, le monologue constituant un forme apprise et artificielle. Volochinov et Bakhtine, sans parler de Vygotski, tirent du dialogue des analyses souvent proches, en dépit d'objets différents (discours intérieur pour Volochinov, dialogisme généralisé pour Bakhtine, ontogenèse de la pensée pour Vygotski, de l'interaction sociale à la construction intérieure) ${ }^{(27)}$.

Comme Jakubinsky, Volochinov prend le dialogue - au sens d'interaction verbale en face à face, ou bien comme dialogue interne ce qui est pour lui l'équivalent de la conscience - pour la seule unité linguistique pertinente. Il l'oppose ainsi à ce qu'il nomme « l'objectivisme abstrait » de Saussure, illustré par l'effort du linguiste pour saisir la permanence des formes du langage organisées en système. Pour Volochinov la linguistique (de l'objectivisme abstrait) se réduit à l'énoncémonologue, contre lequel il part en guerre et auquel il substitue le dialogue.

Qu'il soit représenté par l'échange verbal entre deux personnes ou qu'il soit la manifestation du langage intérieur, le dialogue est imprégné de la réaction à venir d'autrui ou bien se trouve réactif par rapport à la pensée d'autrui ; aucune pensée ne vient donc ex nihilo.

La pensée n'est pas davantage l'expression subjective d'un « moi » expressif :

«Ce n'est pas l'activité verbale qui organise l'expression mais au contraire, c'est l'expression qui organise l'activité mentale, qui la modèle et détermine son orientation ${ }^{(28)}$

Il récuse ainsi autant les thèses du « subjectivisme individualiste » que celles de «l'objectivisme abstrait » au profit d'une troisième voie : l'énonciation (produit de l'acte de parole) ne peut nullement être considérée comme individuelle au sens étroit du terme : selon lui, l'énonciation est de nature sociale ${ }^{(29)}$.

Ou encore :

«L'activité mentale intérieure du sujet constitue, tout autant que l'expression extérieure, un territoire social. De même que tout l'itinéraire qui mène de l'activité mentale (le "contenu à exprimer") à son objectivation externe (1'“énonciation") se situe entièrement en territoire social. ${ }^{(30)}$

C'est à la lumière d'une confrontation féconde avec Benveniste (ce qui prévaut est-il le «territoire social» ou la langue ?) que le dialogisme bakhtinien peut être interprété.

(27) Voir Khyeng, 2003.

(28) V.N. Volochinov, [1929], (1977, traduction Marina Yaguello) : Le marxisme et la philosophie du langage, Paris, Editions de Minuit, pp. 122-123.

(29) Ibid. p. 119.

(30) Ibid. p. 129. 


\subsection{Dialogue et dialogisme}

A la suite de Jacques Brès (2005 : 47-61), on appellera dialogall'échange de paroles avec alternance des sujets parlants : est dialogal ce qui se comprend comme un tour de parole alternant au milieu d'autres répliques. Est dialogique 1'orientation de l'énoncé vers d'autres énoncés : l'énoncé est une réponse à d'autres antérieurs, mais pas nécessairement exprimés, et cette réponse est le fruit d'un dialogue interne à l'énoncé.

D'où une définition de dialogique, à partir des textes de Bakhtine comme « orientation constitutive et au principe de sa production, vers les énoncés réalisés antérieurement sur le même objet de discours et vers la réponse qu'il sollicite » (p. 52).

Ce travail d'élucidation avait été fait de façon pionnière par J. Authier-Revuz (1995, Tome I. 168-172, et 249-255), distinguant trois sortes de dialogisme chez Bakhtine.

Ce qu' elle appelle « dialogisme 1 » (autodialogisme) correspond à son propre champ d'étude pour la réflexivité à l'œuvre dans la langue, c'est le rapport du locuteur avec son propre mot, avec l'opacification que suppose l'arrêt sur ce mot. Chez Bakhtine, il s'agit du constat que le mot choisi par le locuteur lui échappe pourtant, d'une part parce qu'il est traversé d'un discours autre, et qu'il ne se présente jamais comme la propriété du locuteur, que son sens est déjà alourdi des couches de sens précédentes (voir dialogisme 3), mais aussi et surtout parce que le mot (dit, présenté dans le roman) ne peut jamais représenter ce qu'il est chargé de représenter, à savoir le sens. Le locuteur (ou l'auteur de romans) ne peut se mettre à l'extérieur du langage et contempler en position de maîtrise le langage à la fois dans l'acte de dire et d'être représenté. Ce clivage intime du langage est à la base du dialogisme comme «non-un» (J. Authier-Revuz). Pour sa propre théorie, J. AuthierRevuz en tire l'impossibilité d'une maîtrise du sens, ce qui n'invalide pas les tentatives de clarification, de transparence pour soi-même et pour l'autre auxquelles tout locuteur se livre.

Le « dialogisme 2 » correspond au dialogisme interlocutif; il reprend l'idée que tout dialogue est constitué, imprégné, pré-formé dans l'attente de l'autre à qui on s'adresse : c'est l'idée de la «compréhension responsive» (appelant une réponse). On le sait, à l'origine et au principe de l'analyse bakhtinienne se trouve l'affirmation constante que tout énoncé est déjà dialogue, c'est-à-dire non seulement ouverture à la réponse d'autrui, mais écrin, contexte constituant et constitué de la réponse à venir. C'est qu'il n'est aucun énoncé qui ne soit fait de « déjà dit » et en retour, aucun énoncé qui ne soit proféré sans tenir compte de ce qui va s'insérer à sa suite. Tout énoncé se constitue donc en cadre pour l'énoncé qu'il appelle, de même qu'il s'inscrit dans le cadre qui l'a préparé et grâce auquel il sera reçu.

Le « dialogisme 3 », qui correspond au dialogisme interdiscursif, évoque plutôt l'idée qu'il n'y a pas de mot «à soi »; tout discours s'inscrit toujours dans le « déjà dit » des autres discours. J. Authier-Revuz voit là une possible préfiguration de ce qui sera appelé « l'interdiscours » en analyse de discours. On y retrouve en effet la détermination par l'histoire, du discours et du sens.

Du dialogisme bakhtinien, Authier-Revuz retient des formes d'hétérogénéité distinctes : le " discours dans le discours » est cette " hétérogénéité constitutive» issue du dialogisme interne. Mais elle y ajoute une dimension incontournablement métaénonciative : la langue est présente par sa réflexivité, la possibilité lais- 
sée au locuteur de revenir sur son énonciation tout autant qu'à gloser son propre discours.

C'est l'ensemble de ces dimensions qui se retrouvent dans le chantier ouvert des brouillons, où éclate la complexité d'un sujet singulier et collectif, social parce qu'institué par l'école et constitué tout autant par le réel de la langue.

La rature, matérialisée ou non sémiotiquement par une biffure, est l'expérience de ces différentes dimensions, transportée sur un mode dialogique.

\section{Pour une lecture dialogique de l'écriture scolaire}

Je vais prendre maintenant quelques exemples de formes de subjectivité caractéristiques d'une linguistique dialogique à l'école, dans des situations typiquement scolaires.

\subsection{Le dialogisme de la consigne : un dialogisme interlocutif ?}

Une particularité de l'écriture scolaire réside dans le fait que l'énonciation est " provoquée » explicitement par la consigne. Celle-ci peut se présenter de façon diverse, soit formellement par un énoncé, soit par un commentaire oral de celui-ci, assorti d'instructions, d'explications, de conseils, sans qu'il soit toujours possible de démêler ce qui prédomine.

La consigne concentre naturellement diverses formes de dialogisme, dont les manifestations sont souvent accessibles par la reprise des mots de l'énoncé dans le discours du scripteur-élève, en réponse implicite à l'injonction d'écrire contenue dans le libellé.

Cette reprise mérite qu'on s'y arrête : elle diffère en effet de la reprise en modalisation autonymique que l'on trouve dans les dialogues oraux « en face à face », typiques des situations de dialogues didactiques:

\section{EX1 ${ }^{(31)}$}

(329) M. : allez au tableau/ tu vas nous montrer

(330) Séb. : le sujet euh c'est pas/ c'est pas « le film que je », c'est « le film que je regarde $» .$.

(331) $\underline{\mathrm{Cl}}$ : c'est comme en haut maître

(332) M. : c'est comme en haut / «c'est le film que je regarde qui est un western »

Dans l'exemple suivant, ce sont déjà des autonymes qui sont repris en « modalisation autonymique » par l'enseignant :

\section{EX2}

(14) $\underline{\mathrm{M}}$ : d'accord donc si c'est le sujet de «vais » tu fais une flèche...

(15) $\mathrm{Cl}$. : ...une flèche de « je » à « vais »

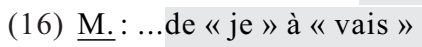

(17) Séb. : le sujet de «possède deux bassins »/ c'est « la piscine où je vais nager»

(18) $\underline{M}$ : « la piscine où je vais nager» oui $\pi /$ vous êtes d'accord $\lambda$

(31) Pour les exemples 1 à 3 : M. = initiale pour Maître. Il s'agissait d'une classe de CM2 en ZEP, à Argenteuil banlieue Nord de Paris. Je remercie l'enseignant, Loïc Martin, de m'avoir autorisée à l'époque (en 1999) à enregistrer son travail avec sa classe. 
Le dernier exemple ci-dessous est incompréhensible si l'on ne sait pas que la formule étrange d'Elodie il y a un sujet dans le sujet résulte d'une invention métalinguistique collective pour reconnaître que la relative - phrase elle-même constituée d'un sujet et d'un verbe - fait partie, dans l'exemple ${ }^{(32)}$ analysé par la classe, du GN sujet :

\section{EX3}

(358)M. [écrivant] : Dans certaines phrases il y a un verbe dans le sujet

(359)Elo. : des fois y a un autre sujet dans le sujet

(360) M. : y a un autre sujet dans le sujet /

[écrivant]: Dans certaines phrases il y a un verbe dans le sujet et un autre sujet dans le sujet $<\mathrm{d}$ 'accord $<$

Ces trois exemples de modalisation autonymique relèvent des formules en «écho » analysées par J. Authier-Revuz comme « dire de X commenté en dialogue ». Elle envisage notamment le cas de " l'enchaînement supporté par deux énonciateurs successifs, d'une énonciation standard de X par L ${ }^{(33)}$ et d'un commentaire par $\mathrm{R}^{(34)}$ de cette énonciation ${ }^{(35)}$. »

Bien que son analyse porte surtout sur le commentaire de R et moins sur la reprise interlocutive, on décèle ici, dans l'énonciation magistrale de reprise, ce fameux « deux en un » du dialogisme, accord fugace entre voix de l'élève et celle de l'enseignant.

Dans les exemples qui suivent, ce dialogisme provient des mots de l'élève et la reprise est d'une autre nature puisqu'elle est surtout scripturale, et s'exerce à partir des mots de la consigne.

\subsection{Les mots du sujet scripteur traversés d' « autre» : deux exemples}

Le fait de répondre à une consigne inscrit cette énonciation en effet dans un double dialogue : avec soi et avec autrui : la mixité de voix énonciatives qui se succèdent ou se substituent l'une à l'autre - que le texte de la consigne soit « absorbé » dans les mots de l'élève ou que les mots du texte de l'élève soient une reprise massive de ceux du texte-princeps - est la règle majeure et assurée du caractère scolaire de ces productions fictives, que l'exercice scolaire dont nous parlons comporte explicitement ou non dans les mots de la consigne la notion de continuité d'un texte à l'autre. Mais rien ne permet aux élèves de savoir comment « greffer » leur propre discours sur une consigne qui est elle-même largement ambiguë, à la fois récit et citation de celui-ci pour s'exhiber comme consigne.

Voici un premier type de dialogisme à partir de la consigne. On s'interrogera sur la forme de reprise dont témoigne le texte d'élève suivant ${ }^{(36)}$.

(32) A savoir : La piscine où je vais nager possède deux bassins.

(33) $\mathrm{L}=$ Locuteur premier

(34) $\mathrm{R}=$ Récepteur

(35) Authier-Revuz, J., 1995 :150-151, (Tome I).

(36) La leçon portait sur le dialogue, à partir du manuel A portée de mots CE2. Je remercie Agathe Desmonts l'enseignante de cette classe de CE2 à Boulogne-Billancourt de m'avoir autorisée à travailler pendant plusieurs séances avec ses élèves de CE2 en décembre 2007. 


\section{EX4}

Consigne :

Voici le début d'une histoire :

Patricia apprend une leçon de géographie. Son petit frère Frédéric s'approche avec un stylo à la main et trace quelques traits sur le cahier. Patricia est désolée. Elle va trouver sa maman.

a) avec un ou plusieurs camarades, écris le dialogue entre ces deux personnages (une vingtaine de lignes)

b) pense à écrire un dénouement à l'histoire.

Charlotte, version 2 :

1 Patricia apprend une leçon de Géographie.

2 «Oh! J'apprendrais jamais cette leçon »! Protesta

3 Patricia.

4 Son petit frère Frédérique [sic] s'approche avec un

5 stylo à la main.

6 « Ga! Ga! J'arrive avec mon stylo magique et

7 je vais faire des petits dessins ! Chantonna Frédérique

La scriptrice répond à la consigne en reprenant exactement (en gris foncé) les mots du texte qui initient l'histoire, soit les deux premières lignes. Cette reprise est-elle à l'identique ? Bien que muette (puisque écrite) cette reprise fonctionne-telle comme les reprises orales en écho étudiées ci-dessus et qui provenaient de l'enseignant?

On observe qu'une reprise ne peut jamais être identique, quand bien même le texte le serait ${ }^{(37)}$, puisque le temps, la situation énonciative diffèrent.

Dans cet exemple, c'est d'abord le présent qui, désactualisé dans la consigne, est narrativisé dès la première phrase (ligne 1 puis lignes 4 et 5). Ainsi modalisée, l'autonymie de cette répétition de segment répond illocutoirement au modèle donné par la consigne. L'appropriation des mots de la consigne par leur reproduction exacte est typiquement scolaire en ce qu'elle est la règle non écrite de la conformité à la demande magistrale (ou extérieure, ou médiée par la consigne), qui se situe moins sur le mode du comme vous dites, que sur celui du : comme vous me dites de dire, la reprise pouvant être lue comme :

«Patricia apprend une leçon de Géographie », comme vous me dites de dire.

Contrairement aux exemples 1 à 3 qui sont des reprises orales en écho, il n'y a pas de rapport immédiat et « audible» entre les segments repris par le scripteur, si ce n'est par la réception de l'ensemble consigne + texte du scripteur qu'en ferait un lecteur tiers, le «troisième dans le dialogue » esquissé par Bakhtine.

Le texte reproduit au présent, cependant, est en conflit avec le passé simple qui affecte uniquement les incises « Protesta » (1.2) et «Chantonna » (1.7).

(37) La nouvelle de Borges dans Fictions qui prétend le Quichotte de Ménard - pourtant identique à celui de Cervantès-, supérieur à celui de son prédécesseur est l'illustration virtuose et littéraire de ce paradoxe. En fait c'est la réception qui ne peut être la même car le temps introduit une différence dans la situation énonciative des sujets et des récepteurs. 
Or celles-ci sont un ajout, en réponse à l'injonction explicite cette fois de l'enseignante, comme le montre le document de la version $1^{\left({ }^{(3)}\right.}$ ci-dessous.

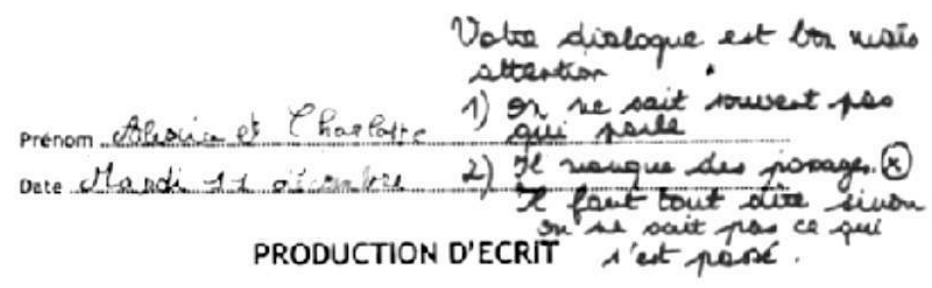

Introduire un dialogue dans le récit

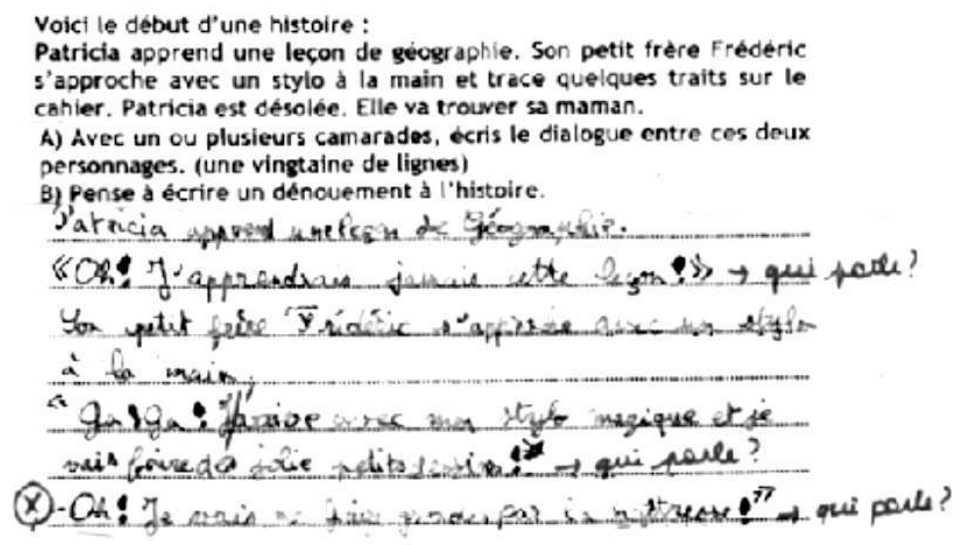

L'hétérogénéité qui en résulte signe le conflit des réponses aux voix interdiscursive et interlocutive contenues dans l'énoncé de la consigne puis dans l'injonction magistrale de la version 1.

Quelle fonction attribuer par ailleurs aux parties dialoguées (zones en gris clair de 1'exemple 4, lignes 2, et lignes 6-7)?

On remarque que ces discours directs agissent ici comme une sorte de glose métadiscursive de ces reprises, mais une glose sans les mots pour le dire; il s'agit plutôt d'une expansion :

4 Son petit frère Frédérique $[\mathrm{sic}]$ s'approche avec un

5 stylo à la main.

6 « Ga! Ga! J'arrive avec mon stylo magique et

7 je vais faire des petits dessins!

y compris sur le mode de la dénégation :

1 Patricia apprend une leçon de Géographie.

2 «Oh! J'apprendrais jamais cette leçon »!...

La reprise en écho traversée « d'autre » est donc à son tour glosée par «l'hétéro-

(38) Cette $1^{\text {re }}$ version a été élaborée à deux. La scriptrice Charlotte est dans les deux cas l'auteur physique de la transcription. 
généité montrée» (J. Authier-Revuz) du discours direct, processus qui enclenche alors le contenu du texte.

\subsection{Dialogisme intertextuel et interdiscursif : quand l'enseignant récrit le texte de l'élève}

Une autre forme de dialogisme s'exerce lorsque l'enseignant retranscrit le texte de l'élève. Dans une classe à double niveau de CM1/CE1 de la banlieue parisienne, les 7 élèves de CM1 de la classe sont invités à faire une réécriture du conte de la Belle et la Bête dont la lecture a été arrêtée à mi-parcours, la fin devant être modifiée. Le scénario de suite de conte, d'abord élaboré en groupes, est rédigé individuellement au long de plusieurs séances. Pour éviter que les élèves ne perdent le fil, l'enseignante ${ }^{(39)}$ décide de transcrire elle-même sur traitement de texte les versions successives de ses élèves ${ }^{(40)}$.

Elle se trouve alors confrontée à la reprise du texte de l'élève en cours de textualisation, s'inscrivant à son tour en « surimpression » du sujet scripteur princeps.

Les dissonances éclatent immédiatement : il est en effet, nous venons de le voir, impossible à un interprète de lire deux fois le même texte avec le même sens. Un sujet autre s'inscrit à la suite du scripteur.

Si nous suivons le texte de Léa, nous voyons combien l'élève est hésitante dans le suivi du matériau graphique, hésitant entre « Belle » (prénom) « la Belle » « la belle » (texte de Léa)

\section{EX5}

\section{Version 3 Etat 2 (version collective transcrite par Lea)}

8 Belle sursauta, elle recula et sentit une main velue...

9 Elle se retourna et vit une Bête par terre.

10 La bête avait une cicatrice était poilue de couleur sombre.

11 le monstre avait des oreilles poilus sur le crane.

12 Il se réveilla. La Belle lui demanda pourquoi il c'était évanouit.

13 Je vais te raconter ce qu'il m'est arrivé. Je faisais la collection de miroirs et un jour

14 j'en ai acheté un. Mais quand j'ai voulu le mettre avec les autres il est tomber

15 et une sorcière est sorti et ma jetée un sort. Tous les ans au Printemps

16 quelqu'un doit trouver une rose jaune. La belle demanda

Cependant, l'enseignante, dans la transcription qu'elle fait, rétablira automatiquement la majuscule pour les deux substantifs, quelle que soit leur position, en s'appuyant sur la norme éditoriale et sans tenir compte des effets de sens possibles du passage de la minuscule à la majuscule. Mais bien plus : la transcription de l'enseignante transforme le texte en l'interprétant, comme le montrent les exemples de « bête » ci-dessous :

(39) Il s'agit d'une enseignante maitre-formateur de banlieue parisienne, expérimentée, plutôt investie dans l'écriture, mais pratiquant une pédagogie " traditionnelle » de classe ordinaire.

(40) L'établissement n'est pas suffisamment équipé pour que les élèves aient chacun un poste. 


\section{EX6 Version 3 Etat 2 (version collective transcrite par Lea)}

[c'est la Bête qui parle à la Belle] :

19 Si tu ny arrive pas tu seras mit à mort et je resterai bête jus'qua

20 se que quelqu un essaye de rompre mon sort

\section{EX7 Version retranscrite par la maîtresse}

20 «Si tu n'y arrives pas tu seras mise à mort et je resterai ainsi jusqu'à ce que

21 quelqu'un rompe mon sort. »

ou encore :

\section{EX8 Lea Version 6 Etat 2}

36 ... Je te remercie Belle sent toi je serais encore bête.

Ce qui donne, revu par la maîtresse :

\section{EX9 Version retranscrite par la maîtresse}

35 "Je te remercie Belle sans toi je serais encore une Bête.»

Le texte reçoit ainsi des déterminations contradictoires, et d'abord au niveau du signifiant graphique, qui entraînent une modification du message (emploi du déterminant indéfini) avant que celui-ci ne soit exprimé jusqu'à son terme.

Or dans le texte du XVIII ${ }^{\mathrm{e}}$ siècle, le nom « bête », contrairement à « belle » toujours en italiques, reste commun, sans majuscules ni italiques, ce qui permettra une équivoque sur le mot et une polysémie sur laquelle joue le texte d'origine :

«mais outre que je suis laid, je n'ai point d'esprit : je sais bien que je ne suis qu'une bête. On n'est pas bête, reprit la belle, quand on n'a point d'esprit : un sot n'a jamais su cela. » ${ }^{(41)}$

Les contemporains ne s'y trompaient pas, et nous avons là un signal d'interprétation du texte qui résulte du co-texte : le petit dialogue didactique qui a précédé le récit du conte a fait apparaître le danger d'avoir trop d'esprit : la Bête est bonne avant tout, et c'est ce qui touche la Belle, séduite et amoureuse avant que ne se produise la métamorphose. Les transcriptions de l'enseignante ont ainsi pour objet de (r)établir un sens univoque sans donner au scripteur la chance de découvrir - pour l'approfondir ou en jouer - l'ambivalence de son propre mot.

Un dernier exemple montre encore la forme de « violence interprétative » exercée sur le texte par le récepteur.

La «moralité » du conte inventée ainsi par Mehdi :

(41) Allera, S. et Reynaud, D., $2002: 95$ 


\section{EX10 Mehdi}

33 Cette histoire montre qu'il ne faut pas faire atantiont a l'apparance

est retranscrite ainsi par l'enseignante :

\section{EX11}

Cette histoire montre qu'il ne faut pas autant se fier à l'apparence

ce qui travestit totalement le sens élaboré par le scripteur.

Il apparaît bien ici à quel point l'énonciation d'un sujet est d'abord une relation et non simplement l'énonciation solipsiste d'un sujet : on voit bien en effet comment pourraient se dessiner les conditions qui feraient du scripteur véritablement un sujet : elles passent par la co-construction d'un point de vue sur le texte qui crée les conditions d'une énonciation véritable. Or, c'est dans la langue que cette construction est possible : la leçon éducative du conte n'est pas donnée seulement par une leçon de morale mais dans l'exploration des différences de forme/sens que chaque scripteur découvre, par l'éclairage dialogique, dans sa propre écriture.

\section{Conclusion}

Que retenir de cette analyse pour le sujet de l'écriture à l'école ? Quels apports en didactique de l'écriture?

Si l'on tient absolument à nommer différentes instances du sujet scripteur présentes virtuellement et (parfois) simultanément dans les écrits fictionnels à l'école, il me semble que l'on pourrait dégager :

- Le scripteur scolaire empirique, dont les affects et la situation individuelle sont évidemment présents à l'horizon, mais ne s'expriment que contenus dans sa situation d'élève : c'est ce sujet qui s'exprime lors des exercices d'auto correction destinées à le faire « revenir sur» son texte, sur le mode :

j'ai réussi versus je n'ai pas réussi,

discours effectué en tant qu'élève, en réponse à l'injonction d'écrire contenue dans la consigne ou le discours magistral.

Il présente une différence avec le sujet méta-discursif ${ }^{(42)}$ qui commente pour lui-même son travail d'écriture sous une forme détachée de la chaîne écrite, mais co-présente dans l'espace scriptural, ainsi qu'on le trouve parfois en marge dans les copies : « alinea » ou « OK», par exemple, car ce dernier exprime ainsi sous leur forme explicite les commentaires prospectifs ou rétrospectifs qu'A. Grésillon (2002) prête au « sujet performatif abstrait » extériorisant la parole intérieure dans l'avant-texte.

- Le sujet de l'écriture, c'est aussi le scripteur divisé des différentes versions : il

(42) Dans un sens large de glose d'un discours, en l'occurrence ici, celui du scripteur après relecture. 
ne lui est pas nécessaire d'effectuer un discours métalinguistique à côté ou en plus de son texte : le langage de la rature est son langage, de même que les signes réflexifs de son activité scripturale : blancs, retours à la ligne, ponctuation et d'une manière générale toutes les "opérations » de réécriture, qu'elles soient visibles dans le même état du texte ou qu'elles résultent d'états successifs de l'élaboration du texte. Sa division est celle de tout scripteur qui est soumis à ce que J. AuthierRevuz (1995) appelle les «non-coïncidences du dire » : non-coïncidence du discours à lui-même, non-coïncidences entre les mots et les choses, des mots à euxmêmes.

Je n'ai exploré qu'une infime partie de cette division intime du langage à laquelle est confronté le scripteur dès l'instant où il s'énonce, en donnant à voir l'extrême richesse du champ qui s'ouvre pour une didactique du sujet énonciateur.

Celle-ci doit s'affranchir auparavant des tentations du psychologisme et de l'artistique.

Etre sujet ce n'est pas exprimer son moi, c'est partager l'exercice de la langue avec d'autres, et donc, toujours mettre en jeu la dialectique subjectivité et collectivité. Ce n'est pas non plus, à mon sens, exactement « être un auteur » ${ }^{(43)}$ au sens artistique mais plutôt au sens auctorial du terme. Le scripteur conquiert son rapport à la langue par l'expérience tout à la fois unique et collective de l'écriture. Cette conquête est rendue difficile par les pesanteurs ou les malentendus d'une certaine tradition scolaire de l'écriture dans laquelle s'inscrivent encore de nombreuses pratiques.

Les «malentendus » inhérents au dialogisme de la consigne que j'ai essayé de décrire, mais aussi les formes du dialogue écrit entre l'enseignant et le scripteur, et leur réception, relèvent d'une prise de conscience progressive que l'énonciation écrite est traversée de dialogismes de plusieurs sortes. Parce que le sujet scolaire de l'écriture est à la fois sujet individuel et collectif, membre d'une communauté discursive fondée sur les échanges et les interactions (travaux de Bernié, Jaubert et Rebière dans ce numéro), le dialogisme interdiscursif, interlocutif s'exerce tout autant que l'autodialogisme. Apprendre à les tenir ensemble relève des apprentissages peu visibles mais indispensables du travail de l'écriture.

(43) Le fait d'être élève entraîne (contraint) la situation d' « auteur », qui n'est pas sans ambiguïté : le scripteur est auteur-en-classe ( auteur $_{1}$, et c'est à ce titre qu'il écrit. Mais il est aussi, en un second sens, « auteur 2 » qui produit/invente, ce qui correspond au locuteur de Ducrot (L) lequel on le sait, peut être saisi alternativement comme locuteur « en tant que tel» ou locuteur « en tant qu'être du monde » $(\lambda)$, c'est-à-dire objet de l'énoncé. 


\section{Références}

AlLERA, S. et REYNAUd, D. (2002) : «La Belle et la Bête. Quatre métamorphoses (1742-1779)» Publications de l'Université Saint-Etienne, collection « Textes et Contre-Textes $\gg \mathrm{n}^{\circ} 2$.

AUTHIER-REVUZ, J. (1995): Ces mots qui ne vont pas de soi : boucles réflexives et non-cö̈ncidences du dire, Tomes I et II, Paris, Larousse, coll. «Sciences du langage $», 869 \mathrm{p}$.

BAKHTINE, M. / Volochinov, V.-N. $(1929,1977)$ : Le marxisme et la philosophie du langage, traduction française de M. Yaguello, 1977, Paris, Minuit, collection «Le sens commun », 233 p.

Benveniste, E. (1966, 1974) : Problèmes de linguistique générale, Paris, Gallimard collection TEL, tomes I et II, $356 \mathrm{p}$. et $286 \mathrm{p}$.

BARRÉ DE MINIAC, Ch. (2000) : Le rapport à l'écriture, aspects théoriques et didactiques, Villeneuve d'Ascq (Nord), Septentrion.

BertuCCI, M.-M. (2007) : «La notion de sujet », in Le Français Aujourd'hui $\mathrm{n}^{\circ} 157$ «Sujet lecteur, sujet scripteur », Paris, Armand-Colin/AFEF, pp. 1118.

BERCHADSKY, J. (1992) : «Le dialogisme», Actes de lecture ${ }^{\circ} 38$ et 39, Association Française pour la Lecture.

BISHOP, M.-F. (2007) : «L'élève sujet dans les textes prescripteurs de l'école primaire » in Le Français Aujourd'hui $\mathrm{n}^{\circ} 157$ « Sujet lecteur, sujet scripteur », Paris, Armand-Colin/AFEF, pp. 19-29.

BORÉ, C. (2010) : Modalités de la fiction dans l'écriture scolaire, Paris, L'Harmattan, collection « Savoir et Formation ».

Bonnet, C., Corblin, C. \& Elalouf, M.-L. (1998) : Les procédés d'écriture chez les élèves de 10 à 13 ans, un stade de développement. Lausanne, Editions LEP (Loisir et Pédagogie).

BRANCA, S.\& GARCIA-DEBANC, Cl. (2007) : «L'avant-texte à l'école primaire : quelques jalons de 1850 à nos jours », Langue Française $\mathrm{N}^{\circ} 155$, pp. 35-50.

BRÈS, J. (2005) : « Savoir de quoi on parle : dialogue, dialogal, dialogique ; dialogisme, polyphonie... » in Dialogisme et polyphonie collection « Approches linguistiques », Actes du colloque de Cerisy-la-Salle, De Boeck-Université, Dunod, Belgique, 47-61.

BRÈS, J. et NOWAKOWSKA, A. (2006) : «Dialogisme : du principe à la matérialité discursive »in Perrin L. (éd.), Le sens et ses voix, Recherches linguistiques 28, Metz : Université de Metz, 21-48.

Bucheton, D. (1995) : Ecritures, Réécritures, récits d'adolescents, Berne, Peter Lang, 296 p.

Bucheton, D. \& Chabanne, J.-Ch., (dir.) (2002) : Parler et écrire pour penser, apprendre et se construire. L'écrit et l'oral réflexifs, P.U.F., $252 \mathrm{p}$.

CHAuvier, S. (2001) : Dire « je », essai sur la subjectivité, Librairie philosophique J. Vrin.

Clanché, P. (1988) : L'enfant écrivain, Paris, Païdos-Le Centurion, 208 p.

DelCAMBRE, I. (2007) : «Du sujet scripteur au sujet didactique » in Le Français 
Aujourd'hui $\mathrm{n}^{\circ} 157$ « Sujet lecteur, sujet scripteur », Paris, Armand-Colin, AFEF, pp. 33-41.

DelCAMBRE, I., REUTER, Y. (2002) : «Images du scripteur et rapport à l'écriture », revue Pratiques n ${ }^{\circ}$ 113-114, Metz, CRESEF, pp. 7-28.

DoqueT-LACOSTE, Cl. (2008) : " Ancrages linguistiques de la didactique de l'écriture à l'école primaire », Congrès Mondial de Linguistique française, pp. 489-504.

DUCROT, O. (1984) : Le dire et le dit, chapitres VII « L'argumentation par autorité » pp. 149-169, et VIII « Esquisse d'une théorie de la polyphonie » pp. 170233, Paris, éditions de Minuit, collection « Propositions », 233 p.

FABRE, Cl. (1982) : « Dans la poubelle de la classe : subjectivité et jeux de langage » La linguistique volume 18, fascicule 2, pp. 99-113.

- (1990) : Les brouillons d'écoliers ou l'entrée dans l'écriture, Grenoble, Céditel /L'Atelier du texte, Université de Grenoble-Stendhal, 233 p.

- (2004) : «Les brouillons et l'école : ce qu'a changé la critique génétique », Le Français Aujourd'hui, n 144 (Boré \& Huynh, éds), AFEF, Armand-Colin, pp. 18-24.

Froment, M. (1988) : Temps et dramatisation dans les récits écrits d'élèves de 5ème, Louvain-la Neuve, Peeters, $264 \mathrm{p}$.

KYHENG, R. (2003) : «Aux origines du principe dialogique. L'étude de Jakubinskij : une présentation critique ». In Texto ! Textes et cultures [en ligne], décembre 2003, vol. VIII, $\mathrm{n}^{\circ}$ 4.Disponible sur : ttp://www.revuetexto.net/Inedits/Kyheng/Kyheng_Jakubinskij.html.

LAFONT-TERRANOVA, J. (2009) : Se construire à l'école comme sujet-écrivant PUN, coll. Diptyque.

ONO, A. (2007) : La notion d'énonciation chez Emile Benveniste, préface de Michel Arrivé, postface de Claudine Normand., Limoges, éditions LambertLucas.

LEBRAVE, J.-L. (1983) : «Lecture et analyse des brouillons », Langages, 63, 11 23.

Petitjean, A. (1999) : «Un siècle d'enseignement de la composition française ou de la rédaction au primaire (1882-1995) » in Petitjean, A. \& Privat, J.-M., (dir.), 1999 : Histoire de l'enseignement du français et textes officiels, Actes du colloque de Metz, Numéro 9, CELTED, Université de Metz, 83-116.

PLANE, S. (dir.) (2003) : «L'écriture et son apprentissage à l'école primaire », $R e$ pères, $\mathrm{N}^{\circ} 24-25$, Paris, INRP.

- (2005) : «Fiction, utopie et représentation du monde et de l'école dans des écrits sur le collège rédigés par des élèves en difficulté », Langage et Société $\mathrm{n}^{\circ} 111$, L'institution pédagogique, l'écrit et le sujet en formation Maison des Sciences de 1'homme, Paris, 119-148.

SCHEPENS, Ph. (1999) : Linguistique dialogique et psychanalyse, Presses universitaires franc-comtoises, collection Annales Littéraires.

TAUVERON, C. (2007) : «Le texte singulier de l'élève et la question du sujet-scripteur », in Le Français Aujourd'hui n 157 « Sujet lecteur, sujet scripteur », Paris, Armand-Colin/AFEF, pp. 75-82. 